PROCEEDINGS OF THE

AMERICAN MATHEMATICAL SOCIETY

Volume 138, Number 10, October 2010, Pages 3415-3424

S 0002-9939(2010)10243-5

Article electronically published on June 4, 2010

\title{
THE GABRIEL-ROITER SUBMODULES OF SIMPLE HOMOGENEOUS MODULES
}

\author{
BO CHEN
}

(Communicated by Birge Huisgen-Zimmermann)

Dedicated to my wife, Qi, and my twin daughters, Yining and Yimeng

\begin{abstract}
Let $\Lambda$ be a connected tame hereditary algebra over an algebraically closed field. We show that if $\Lambda=k Q$ is of type $\widetilde{\mathbb{A}}_{n}, \widetilde{\mathbb{D}}_{n}, \widetilde{\mathbb{E}}_{6}$ or $\widetilde{\mathbb{E}}_{7}$, then every Gabriel-Roiter submodule of a quasi-simple module of rank 1 (i.e. a simple homogeneous module) has defect - 1 . In particular, any Gabriel-Roiter submodule of a simple homogeneous module yields a Kronecker pair, and thus induces a full exact embedding of the category $\bmod k \widetilde{\mathbb{A}}_{1}$ into $\bmod \Lambda$, where $\widetilde{\mathbb{A}}_{1}$ is the Kronecker quiver. Consequently, we obtain that all quasi-simple modules are Gabriel-Roiter factor modules.
\end{abstract}

\section{Introduction AND MAIN RESUlts}

Throughout the paper, we assume that $k$ is an algebraically closed field. By algebras, we mean finite dimensional basic connected $k$-algebras, unless stated otherwise. The modules over an algebra are always assumed to be finitely generated left modules. We denote by $|M|$ the length of a module $M$. The symbol $\subset$ is used to denote proper inclusion.

The Gabriel-Roiter measure of an Artin algebra is a combinatorial invariant that was used by Gabriel and Roiter in the 1970s in the proof of the first Brauer-Thrall conjecture, dealing with algebras of finite representation type (see [7 for example). Several years ago, Ringel has demonstrated in [8, 9] its relevance for the study of algebras of infinite representation type. Let $\Lambda$ be an Artin algebra. The GabrielRoiter (GR for short) measure $\mu(M)$ for a $\Lambda$-module $M$ was defined (see [9]) by induction as follows:

$$
\mu(M)= \begin{cases}0, & M=0 ; \\ \max _{N \subset M}\{\mu(N)\} & \text { if } M \text { is decomposable; } \\ \max _{N \subset M}\{\mu(N)\}+\frac{1}{2^{|M|}} & \text { if } M \text { is indecomposable. }\end{cases}
$$

The so-called Gabriel-Roiter submodules of an indecomposable module are defined to be the indecomposable proper submodules with maximal measure. An inclusion $X \subset Y$ with $X$ and $Y$ indecomposable is called a Gabriel-Roiter inclusion if $X$ is a Gabriel-Roiter submodule of $Y$. The factor module $Y / X$ of a GR inclusion $X \subset Y$

Received by the editors October 7, 2008 and, in revised form, August 7, 2009 and September 28, 2009.

2010 Mathematics Subject Classification. Primary 16G20, 16G70.

Key words and phrases. Tame hereditary algebras, simple homogeneous modules, defect, Gabriel-Roiter measure.

(C)2010 American Mathematical Society
Reverts to public domain 28 years from publication 3415 
is called a Gabriel-Roiter factor. It was proved in [8] that a Gabriel-Roiter factor is indecomposable, which gives the first proof that every indecomposable module is an extension of two indecomposable ones.

Let $\Lambda$ be a tame hereditary algebra, i.e. a path algebra $\Lambda=k Q$ with $Q$ a quiver of type $\widetilde{\mathbb{A}}_{n}, \widetilde{\mathbb{D}}_{n}, \widetilde{\mathbb{E}}_{6}, \widetilde{\mathbb{E}}_{7}$ or $\widetilde{\mathbb{E}}_{8}$ (for a reference, see [5] for example). A quasisimple module is an indecomposable regular module such that the Auslander-Reiten sequence starting with it has indecomposable middle term. Each indecomposable regular $\Lambda$-module $M$ can be uniquely written as $X[t]$ for some quasi-simple module $X=X[1]$ and some natural number $t$, where $t$ is the length of the unique sequence of irreducible monomorphisms $X=X[1] \rightarrow X[2] \rightarrow \cdots \rightarrow X[t] \cong M$. The rank of a quasi-simple module $X$ over $\Lambda$ is the minimal natural number $r$ such that $\tau^{r} X \cong X$, where $\tau$ is the Auslander-Reiten translation. We use $H_{i}$, instead of $X[i]$, if $X$ is a quasi-simple module of rank 1 , and in this case $H_{1}$ is called a simple homogeneous module. Let $\delta$ be the minimal radical vector, which corresponds to the minimal positive imaginary root of the corresponding affine Lie algebra. Then the dimension vector of $X[r]$ is $\delta$ for each quasi-simple module $X$ of rank $r$. It has been shown that the Gabriel-Roiter measures of indecomposable modules with dimension vector $\delta$ play an important role when comparing (computing) the measures of regular modules (see [4], or Proposition 2.5 below). Thus it is important to know the Gabriel-Roiter measures of these indecomposable modules.

We first study the Gabriel-Roiter submodules of simple homogeneous modules. We also denote by $\delta$ the well-known defect function (see section 2.2 for details).

Theorem. Let $\Lambda=k Q$ be a tame hereditary algebra of type $\widetilde{\mathbb{A}}_{n}, \widetilde{\mathbb{D}}_{n}, \widetilde{\mathbb{E}}_{6}$ or $\widetilde{\mathbb{E}}_{7}$. Assume that $X \subset H_{1}$ is a Gabriel-Roiter inclusion with $H_{1}$ a simple homogeneous module. Then $\delta(X)=-1$.

Under the assumption of the theorem, we may conclude that $\left(H_{1} / X, X\right)$ is a Kronecker pair, i.e. $X$ and $H_{1} / X$ are orthogonal exceptional modules with $\operatorname{dimExt}{ }^{1}\left(H_{1} / X, X\right)=2$. Let $\mathcal{A}$ be the full subcategory of $\bmod \Lambda$ with filtrations with factors $X$ and $H_{1} / X$. Then $\mathcal{A}$ is equivalent to the category of Kronecker modules. It follows that starting with one simple homogeneous module $H_{1}$, any GR submodule yields a Kronecker pair and thus the whole one-parameter family of simple homogeneous modules.

In the proof of the theorem, we will see why the $\widetilde{\mathbb{E}}_{8}$ case is excluded. Namely, the proof that will be presented depends on the $\tau$-orbit of the preprojective modules of defect -1 . For the $\widetilde{\mathbb{E}}_{8}$ case there is only one such $\tau$-orbit. However, for the other cases, there are at least two.

As an application of the theorem, we have the following proposition, which concerns the GR measures of some indecomposable regular modules with dimension vector $\delta$ :

Proposition. Let $\Lambda$ be a tame hereditary algebra of type $\widetilde{\mathbb{A}}_{n}, \widetilde{\mathbb{D}}_{n}, \widetilde{\mathbb{E}}_{6}$ or $\widetilde{\mathbb{E}}_{7}$, and let $X_{1}$ be a quasi-simple module of rank $r>1$ and $X_{i}=\tau^{-(i-1)} X_{1}$. Then there exist some $1 \leq j \leq r$ such that $\mu\left(X_{j}[r]\right) \geq \mu\left(H_{1}\right)$.

This proposition tells us that given a regular tube of rank $r>1$ over a tame hereditary algebra which is not of $\widetilde{\mathbb{E}}_{8}$ type, there is always a quasi-simple module $X$ such that $\mu(X[r]) \geq \mu\left(H_{1}\right)$. However, there may not exist a quasi-simple module $X$ with $\mu(X[r])<\mu\left(H_{1}\right)$. For example, $\Lambda=k \widetilde{\mathbb{A}}_{n}$ with sink-source orientation (see $[3$ for details). 
The Auslander-Reiten sequences ending at Gabriel-Roiter factors were studied in [1, 2] for representation-finite hereditary algebra and tame hereditary algebras, respectively. Most of these Auslander-Reiten sequences have indecomposable middle terms. Assume that $\Lambda$ is a representation-infinite hereditary algebra. A regular Gabriel-Roiter factor must be a quasi-simple module since an irreducible map to a Gabriel-Roiter factor is always an epimorphism. However, it is not known yet if every quasi-simple module is a Gabriel-Roiter factor. Using the above proposition, we can answer the question for tame cases as follows:

Corollary. Let $\Lambda=k Q$ be a tame hereditary algebra of type $\widetilde{\mathbb{A}}_{n}, \widetilde{\mathbb{D}}_{n}, \widetilde{\mathbb{E}}_{6}$ or $\widetilde{\mathbb{E}}_{7}$. Then each quasi-simple module $X$ is a Gabriel-Roiter factor and there are, up to isomorphism, infinitely many Gabriel-Roiter inclusions with factor $X$.

\section{Preliminaries And KNOWn Results}

2.1. General results on Gabriel-Roiter measures. We first recall some results on Gabriel-Roiter measures. The following property follows directly from the definition.

Lemma 2.1. Let $\Lambda$ be an Artin algebra and $X, Y$ and $Z$ be indecomposable $\Lambda$ modules.

(1) If $X$ is a proper submodule of $Y$, then $\mu(X)<\mu(Y)$.

(2) If $\mu(X)<\mu(Y)<\mu(Z)$ and $X$ is a GR submodule of $Z$, then $|Y|>|Z|$.

The following Main Property can be found in [8].

Main Property. Let $\Lambda$ be an Artin algebra and $X, Y_{1}, \cdots, Y_{t}$ be indecomposable modules. Assume that there is a monomorphism $f: X \longrightarrow \bigoplus_{i=1}^{t} Y_{i}$. Then

(1) $\mu(X) \leq \max \left\{\mu\left(Y_{i}\right)\right\}$.

(2) If $\mu(X)=\max \left\{\mu\left(Y_{i}\right)\right\}$, then $f$ splits.

2.2. Defect function. Let $\Lambda=k Q$ be a tame hereditary algebra. We quickly recall some preliminaries and refer to [5] for details and non-explained notions.

We denote by $\delta=\left(\delta_{i}\right)$ the minimal dimension vector such that $\langle\delta, \delta\rangle=0$, where $\langle-,-\rangle$ is the associated Tits form given by

$$
\langle\underline{\operatorname{dim}} X, \underline{\operatorname{dim}} Y\rangle=\operatorname{dimHom}(X, Y)-\operatorname{dimExt}^{1}(X, Y)
$$

for $\Lambda$-modules $X, Y$, where $\underline{\operatorname{dim}}$ denotes the dimension vector. The defect of a module $X$ is defined to be $\langle\delta, \underline{\operatorname{dim}} X\rangle=-\langle\underline{\operatorname{dim}} X, \delta\rangle$. We thus get a defect function, which is also denoted by $\delta: \delta(X)=\langle\delta, \underline{\operatorname{dim} X}\rangle$. It is well-known that an indecomposable $\Lambda$-module $X$ is preprojective (respectively, regular, preinjective) if and only if $\delta(X)<0$ (respectively, $=0,>0$ ). Note that the defect function is a constant on each $\tau$-orbit, and for an indecomposable projective module $P_{i}$,

$$
\delta\left(P_{i}\right)=-\left\langle\underline{\operatorname{dim}} P_{i}, \underline{\operatorname{dim}} H_{1}\right\rangle=-\operatorname{dimHom}\left(P_{i}, H_{1}\right)=-\left(\underline{\operatorname{dim}} H_{1}\right)_{i}=-\delta_{i} .
$$

Using the defect function, we may easily get the following consequence for tame hereditary aglebras.

Lemma 2.2. (1) An indecomposable preprojective module of defect -1 has no proper preprojective factor.

(2) Every nonzero homomorphism from an indecomposable preprojective module of defect -1 to a quasi-simple module is either injective or surjective. 
Proof. We show (1), and (2) follows directly from (1). Let $X$ be an indecomposable preprojective module of defect $\delta(X)=-1$, and let $X \stackrel{f}{\rightarrow} Y$ be a proper epimorphism with $Y$ indecomposable preprojective. We denote by $K$ the kernel of $f$. Then $\delta(K)=\delta(X)-\delta(Y) \geq 0$ since $\delta(X)=-1$ and $\delta(Y)<0$. But $K$ is a submodule of $X$, which is thus preprojective and $\delta(K)<0$, a contradiction.

2.3. The Gabriel-Roiter measure for tame hereditary algebras. From now on, we fix a tame hereditary algebra $\Lambda=k Q$. If $X$ is a quasi-simple module of rank $r$, then $\underline{\operatorname{dim}} X[r]=\delta$. It has been shown in 4] (for the $\widetilde{\mathbb{A}}_{n}$ case, also see 3]) that the Gabriel-Roiter measures of indecomposable modules with dimension vector $\delta$ play an important role when comparing the measures of regular modules (Proposition 2.5 below).

The following propositions can be used to compute (or compare) the GabrielRoiter measures of $\Lambda$-modules. We refer to [4] (for the $\widetilde{\mathbb{A}}_{n}$ case, also see [3]) for the proofs and details.

Proposition 2.3. Let $H_{1}$ be a simple homogeneous module. Then $\mu\left(H_{1}\right)>\mu(M)$ for each indecomposable preprojective module $M$.

As a direct consequence of the above proposition, we have

Proposition 2.4. Let $H_{1} \rightarrow H_{2} \rightarrow H_{3} \rightarrow \cdots$ be a sequence of irreducible monomorphisms with $H_{1}$ a simple homogeneous module. Then for each $i \geq 2, H_{i}$ contains $H_{i-1}$ as the unique GR submodule.

The following proposition is very useful when comparing the Gabriel-Roiter measures of regular modules.

Proposition 2.5. Let $X$ be a quasi-simple module of rank $r>1$.

(1) If $\mu(X[r]) \geq \mu\left(H_{1}\right)$, then $\mu(X[j])>\mu\left(H_{i}\right)$ for all $j>r, i \geq 1$. In this case, $X[j]$ is, up to isomorphism, the unique Gabriel-Roiter submodule of $X[j+1]$ for each $j \geq r$.

(2) If $\mu(X[r])<\mu\left(H_{1}\right)$, then $\mu(X[j])<\mu\left(H_{1}\right)$ for all $j \geq 1$.

\section{Proof of the Theorem}

The main aim of this section is to prove the following theorem:

Theorem. Let $\Lambda=k Q$ be a tame hereditary algebra of type $\widetilde{\mathbb{A}}_{n}, \widetilde{\mathbb{D}}_{n}, \widetilde{\mathbb{E}}_{6}$ or $\widetilde{\mathbb{E}}_{7}$. Assume that $X \subset H_{1}$ is a Gabriel-Roiter inclusion with $H_{1}$ a simple homogeneous module. Then $\delta(X)=-1$.

We first give a generalized version of Lemma 4.1 in 6 , which says that if $\Lambda$ is a hereditary algebra and $X, Y$ are indecomposable $\Lambda$-modules with $\operatorname{Ext}^{1}(X, Y)=0$, then any non-zero homomorphism from $Y$ to $X$ is either injective or surjective. For a natural number $m$, we denote by $[1, m]$ the set $\{1,2,3, \cdots, m\}$.

Lemma 3.1. Let $\Lambda$ be a hereditary algebra and $X, X_{1}, \cdots, X_{m}$ be indecomposable $\Lambda$-modules such that $\operatorname{Hom}\left(X_{i}, X_{j}\right)=0$ for all $i \neq j$. If $\operatorname{Ext}^{1}\left(\bigoplus_{i \in[1, m]} X_{i}, X\right)=$ 0 and $f: X \rightarrow \bigoplus_{i \in[1, m]} X_{i}$ is a nonzero homomorphism such that $\operatorname{im} f$ is not contained in any direct sum of $m-1$ terms, then $f$ is either an epimorphism or a monomorphism. 
Proof. The proof is similar to the original one given in [6]. Assume that $f$ is such a map as in the assumption, and that $f$ is neither injective nor surjective. Denote by $U$ the image of $f$ and by $C$ the cokernel of $f$. Let $\iota$ be the inclusion $U \rightarrow \bigoplus X_{i}$. Since $\Lambda$ is hereditary, the induced map $\operatorname{Ext}^{1}(C, f): \operatorname{Ext}^{1}(C, X) \rightarrow \operatorname{Ext}^{1}(C, U)$ is surjective. Thus there exists a commutative diagram:

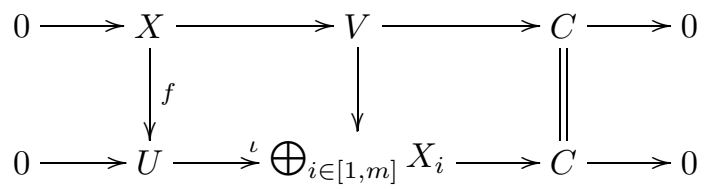

Therefore, we obtain a short exact sequence

$$
0 \rightarrow X \rightarrow U \oplus V \rightarrow \bigoplus_{i \in[1, m]} X_{i} \rightarrow 0 .
$$

We claim that the sequence does not split and thus get a contradiction. Namely, if it splits, then $U$ is isomorphic to $\bigoplus_{j \in J} X_{j}$ for some $J \subset[1, m]$ by the Krull-RemakSchmidt Theorem. Let $t \in[1, m] \backslash J$, and let $\pi$ be the canonical projection $\bigoplus_{i \in[1, m]} X_{i} \rightarrow X_{t}$. Since $\operatorname{Hom}\left(X_{i}, X_{j}\right)=0$ for all $i \neq j$, the restriction $\left.\pi\right|_{U}=\pi \iota$ is zero. Thus $U$ is contained in $\operatorname{ker} \pi=\bigoplus_{i \neq t} X_{i}$. This contradicts the assumption on $f$. We are done.

Proof of the Theorem. For the $\widetilde{\mathbb{A}}_{n}$ cases, this is clear since any indecomposable preprojective module has defect -1 .

Now we consider the $\widetilde{\mathbb{D}}_{n}$ case. Notice that in this case the defect of an indecomposable preprojective module is either -1 or -2 . Assume that $\delta(X)=-2$ and consider the following full subquiver of the preprojective component of the Auslander-Reiten quiver:

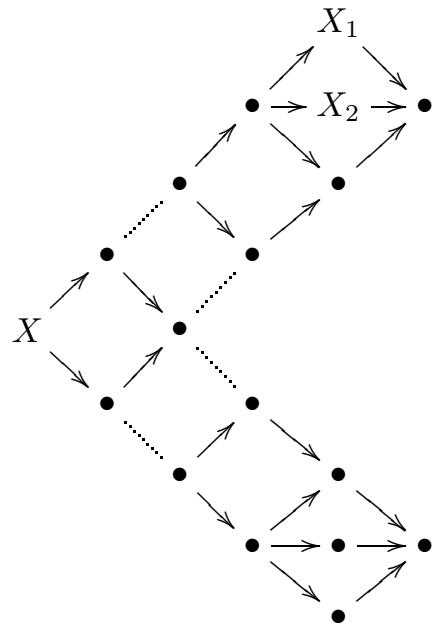

Note that both $X_{1}$ and $X_{2}$ are of defect -1 . Since there is a sectional path from $X$ to $X_{1}$, we get $\operatorname{Ext}^{1}\left(X_{1}, X\right)=0$ and $\operatorname{dimHom}\left(X, X_{1}\right)=1$. The same is true for $X_{2}$. Let $f_{i}: X \rightarrow X_{i}$ be a nonzero homomorphism for $i=1,2$. Then $f_{i}$ is either a monomorphism or an epimorphism by Lemma 3.1 (or the original version). Since $\operatorname{Ext}^{1}\left(X_{1} \oplus X_{2}, X\right)=0$ and the map $X \stackrel{f=\left(f_{1}, f_{2}\right)^{\perp}}{\longrightarrow} X_{1} \oplus X_{2}$ satisfies the 
assumption in Lemma 3.1, it is thus either an epimorphism or a monomorphism. Assume that it is an epimorphism and denote by $K$ the kernel. Then $\delta(K)=$ $\delta(X)-\delta\left(X_{1} \oplus X_{2}\right)=0$ since $\delta(X)=-2$ and $\delta\left(X_{1}\right)=\delta\left(X_{2}\right)=-1$. This implies that $K$ has to be zero, and therefore $f$ is a monomorphism. Thus the Main Property implies that $\mu(X)<\max \left\{\mu\left(X_{1}\right), \mu\left(X_{2}\right)\right\}$. Without loss of generality, we assume that $\mu\left(X_{1}\right)=\max \left\{\mu\left(X_{1}\right), \mu\left(X_{2}\right)\right\}$.

Denote by $X=Y_{s} \rightarrow \cdots \rightarrow Y_{1} \rightarrow X_{1}$ the sectional path from $X$ to $X_{1}$. If both $\tau^{s} X_{1}$ and $\tau^{s} X_{2}$ are non-zero, then there is a sectional path from $\tau^{s} X_{2}$ (respectively $\tau^{s} X_{1}$ ) to $X$. An easy calculation shows that

$$
|X|-\left|X_{1}\right|= \begin{cases}\left|\tau^{s} X_{2}\right| & \text { if } s \text { is even; } \\ \left|\tau^{s} X_{1}\right| & \text { if } s \text { is odd. }\end{cases}
$$

In any case, we get $|X|-\left|X_{1}\right|>0$. Therefore, $f_{1}$ has to be an epimorphism. Thus $\left|X_{1}\right|<|X|<\left|H_{1}\right|$. If one of $\tau^{s} X_{1}$ and $\tau^{s} X_{2}$ is zero (this implies that formally, $X_{1}$ is not far away from projective modules in the Auslander-Reiten quiver), one can thus calculate the possible dimension vectors of $X_{1}$, i.e. the possible dimensions of the Hom-spaces between the projective modules and $X_{1}$, via the paths in the preprojective component, and get that $\underline{\operatorname{dim}} X_{1}<\underline{\operatorname{dim}} H_{1}=\delta$. (In fact, it is sufficient to find one indecomposable projective module $P_{i}$ such that $\left.\operatorname{dimHom}\left(P_{i}, X_{1}\right)=\left(\underline{\operatorname{dim}} X_{1}\right)_{i}<\delta_{i}=\left(\underline{\operatorname{dim}} H_{1}\right)_{i}.\right)$

In each case, we get that $\underline{\operatorname{dim}} X_{1}<\underline{\operatorname{dim}} H_{1}$. Thus there is always a monomorphism from $X_{1}$ to $H_{1}$ by Lemma 2.2, It follows that $\mu\left(X_{1}\right)<\mu\left(H_{1}\right)$. It turns out that $\mu(X)<\max \left\{\mu\left(X_{1}\right), \mu\left(X_{2}\right)\right\}=\mu\left(X_{1}\right)<\mu\left(H_{1}\right)$. Therefore, we obtain a contradiction since $X \subset H_{1}$ is a Gabriel-Roiter inclusion.

The proof of the $\widetilde{\mathbb{D}}_{n}$ case provides an idea of how to prove the $\widetilde{\mathbb{E}}_{6}$ and $\widetilde{\mathbb{E}}_{7}$ cases. We give the main steps under the assumption that $X$ is a Gabriel-Roiter submodule of $H_{1}$ with defect $\delta(X) \neq-1$.

Step 1. On each full sectional path starting with $X$ in the preprojective component, we select (at most) one indecomposable module $X_{i}$ that lies on the boundary of the component; i.e. the middle term of the Auslander-Reiten sequence ending at $X_{i}$ is indecomposable. Then clearly, the relations satisfy $\operatorname{Hom}\left(X_{i}, X_{j}\right)=0$ for all $i \neq j, \operatorname{Ext}^{1}\left(X_{i}, X\right)=0$ and $\operatorname{dimHom}\left(X, X_{i}\right)=1$.

Step 2. We may select those $X_{i}$ such that $\sum_{i} \delta\left(X_{i}\right)=\delta(X)$. Let $f_{i}: X \rightarrow X_{i}$ be a nonzero homomorphism and $f=\left(f_{i}\right)_{i}^{\perp}: X \rightarrow \bigoplus X_{i}$. Then $f$ is either injective or surjective by Lemma 3.1. However, it cannot be an epimorphism since $\delta(X)=\sum_{i} \delta\left(X_{i}\right)$. Thus by the Main Property, $\mu(X)<\max \left\{\mu\left(X_{i}\right)\right\}$, say $\mu\left(X_{1}\right)$ is the maximum measure.

Step 3. If $\delta\left(X_{1}\right) \neq-1$, we may repeat the above steps for $X_{1}$, and obtain an indecomposable preprojective module $M$ with $\delta(M)=-1$ and $\mu(X)<$ $\mu\left(X_{1}\right)<\mu(M)$. If $\delta\left(X_{1}\right)=-1$, set $X_{1}=M$.

Step 4. We may show step by step that the dimensional vector of $M$ is smaller than $\delta$ in the following two ways: either there is an epimorphism from $X$ to $M$, or there exists an indecomposable projective module $P_{t}$ with defect $s$, such that $(\underline{\operatorname{dim}} M)_{t}=\operatorname{dimHom}\left(P_{t}, M\right)<-s=\delta_{t}$.

Step 5. Finally, since $|M|<\left|H_{1}\right|$ and $\delta(M)=-1$, we may obtain a monomorphism from $M$ to $H_{1}$ by Lemma 2.2. Thus we get $\mu(X)<\mu(M)<\mu\left(H_{1}\right)$, which is a contradiction. 
In the following tables, we indicate the selection of such modules $X_{i}$ for a given $X$. There, $\star$ and $\star_{1}$ denote the modules selected for $X$ with $\delta(\star)=-1$ and $\delta\left(\star_{1}\right) \neq-1$. We use $\star_{2}$ to denote the modules selected for $\star_{1}$ in case $\mu\left(\star_{1}\right)=\max \left\{\mu(\star), \mu\left(\star_{1}\right)\right\}$. The black bullets indicate the modules with 3 arrows starting with those in the preprojective component of the Auslander-Reiten quiver.

For the $\widetilde{\mathbb{E}}_{6}$ case, there are two possibilities:

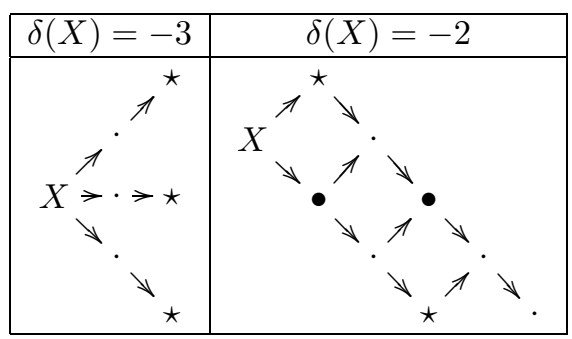

For the $\widetilde{\mathbb{E}}_{7}$ case, there are four possibilities:

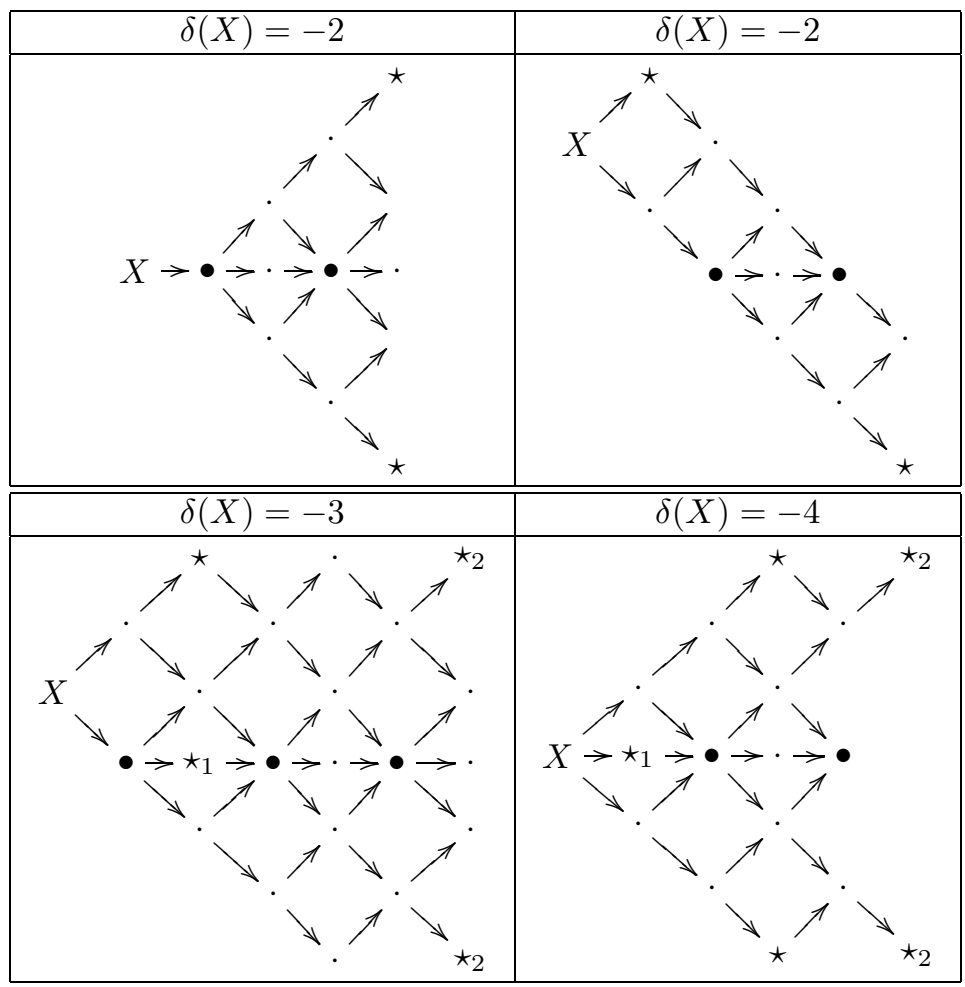

The proof is completed.

Remark. As mentioned in the Introduction, the method in the proof of the Theorem cannot be applied to the $\widetilde{\mathbb{E}}_{8}$ case since there is only one $\tau$-orbit of defect -1 . Thus it is not known at the moment if the theorem holds for the $\widetilde{\mathbb{E}}_{8}$ case. 


\section{KRONECKER PAIRS}

Let $\Lambda$ be a hereditary algebra over an algebraically closed field. An indecomposable $\Lambda$-module $M$ is exceptional if $\operatorname{Ext}^{1}(M, M)=0$. A pair of indecomposable modules $(Y, X)$ is an orthogonal exceptional pair if $X$ and $Y$ are both exceptional modules, and $\operatorname{Hom}(X, Y)=\operatorname{Hom}(Y, X)=0=\operatorname{Ext}^{1}(X, Y)$. In [1], we have shown that for a representation-finite hereditary algebra over an algebraically closed field, each GR inclusion yields a Schofield sequence; i.e. if $X$ is a GR submodule of $M$, then $(M / X, X)$ is an orthogonal exceptional pair. In this case $\operatorname{dimExt}^{1}(M / X, X)=1$.

This can be in some sense generalized to tame hereditary algebras. A pair of indecomposable modules $(Y, X)$ is called a Kronecker pair if $(Y, X)$ is an orthogonal exceptional pair with $\operatorname{dimExt}^{1}(Y, X)=2$. We fix a tame hereditary algebra $\Lambda$.

Proposition 4.1. Let $H_{1}$ be a simple homogeneous $\Lambda$-module and $X$ a submodule of defect -1 . Then $\left(H_{1} / X, X\right)$ is a Kronecker pair.

Proof. Since $H_{1}$ contains no proper regular submodules and since the defect $\delta(X)=-1$, we see that $X$ is an indecomposable preprojective module. It follows that $\operatorname{dimHom}\left(X, H_{1}\right)=1$. Using the Auslander-Reiten formula, we obtain $\operatorname{dimExt}^{1}\left(H_{1}, X\right)=\operatorname{dimHom}\left(X, \tau H_{1}\right)=\operatorname{dimHom}\left(X, H_{1}\right)=1$. Let $\epsilon: 0 \rightarrow$ $X \rightarrow H_{1} \rightarrow H_{1} / X \rightarrow 0$ be the canonical short exact sequence. Then $\delta\left(H_{1} / X\right)=$ $\delta\left(H_{1}\right)-\delta(X)=1$. By duality, $H_{1} / X$ is indecomposable preinjective and $\operatorname{dimHom}\left(H_{1}, H_{1} / X\right)=1$. Thus both $X$ and $H_{1} / X$ are exceptional. Applying $\operatorname{Hom}(X,-)$ to $\epsilon$, we obtain the exact sequence

$$
0 \rightarrow \operatorname{Hom}(X, X) \rightarrow \operatorname{Hom}\left(X, H_{1}\right) \rightarrow \operatorname{Hom}\left(X, H_{1} / X\right) \rightarrow \operatorname{Ext}^{1}(X, X)=0 .
$$

Thus $\operatorname{Hom}\left(X, H_{1} / X\right)=0$ and $\left(H_{1} / X, X\right)$ is an orthogonal exceptional pair. Applying $\operatorname{Hom}(-, X)$ to $\epsilon$, we see that

$$
\begin{aligned}
0=\operatorname{Hom}\left(H_{1}, X\right) \rightarrow \operatorname{Hom}(X, X) \rightarrow \operatorname{Ext}^{1}\left(H_{1} / X, X\right) & \\
& \rightarrow \operatorname{Ext}^{1}\left(H_{1}, X\right) \rightarrow \operatorname{Ext}^{1}(X, X)=0 .
\end{aligned}
$$

Therefore, $\operatorname{dimExt}^{1}\left(H_{1} / X, X\right)=2$ and $\left(H_{1} / X, X\right)$ is a Kronecker pair.

One of the most important properties of a tame hereditary algebra $\Lambda$ is the fact that there are full exact subcategories, which are equivalent to the category of modules over the Kronecker algebra $k \widetilde{\mathbb{A}}_{1}$. Thus our theorem shows that (at least for the $\widetilde{\mathbb{A}}_{n}, \widetilde{\mathbb{D}}_{n}, \widetilde{\mathbb{E}}_{6}$, and $\widetilde{\mathbb{E}}_{7}$ cases) any GR submodule of a simple homogeneous module yields a Kronecker pair and thus induces a full exact embedding of the category of Kronecker modules into $\bmod \Lambda$.

\section{Regular Gabriel-Roiter factors}

Let $\Lambda=k Q$ be a tame hereditary algebra. We have seen in Proposition 2.5 that the GR measures of the indecomposable modules with dimension vector $\delta$ play an important role when comparing the GR measures of regular modules. Thus, given a quasi-simple module $X$ of rank $r>1$, we want to know if $\mu(X[r]) \geq \mu\left(H_{1}\right)$ (or $\left.\mu(X[r])<\mu\left(H_{1}\right)\right)$. However, there may not exist a quasi-simple module of rank $r>1$ such that $\mu(X[r])<\mu\left(H_{1}\right)$. For example, $Q$ is a quiver of type $\widetilde{\mathbb{A}}_{n}$ with sink-source orientation (radical-square zero) (see [3] for details). In [3], it has been shown that given a regular tube of rank $r>1$ over $\Lambda=k \widetilde{\mathbb{A}}_{n}$, there always exists a 
quasi-simple $X$ such that $\mu(X[r]) \geq \mu\left(H_{1}\right)$. This can be generalized to the $\widetilde{\mathbb{D}}_{n}, \widetilde{\mathbb{E}}_{6}$ and $\widetilde{\mathbb{E}}_{7}$ cases since the main thing is that $\delta(Y)=-1$ for a Gabriel-Roiter submodule $Y$ of $H_{1}$.

Proposition. Let $\Lambda$ be a tame hereditary algebra of type $\widetilde{\mathbb{A}}_{n}, \widetilde{\mathbb{D}}_{n}, \widetilde{\mathbb{E}}_{6}$ or $\widetilde{\mathbb{E}}_{7}$, and let $X_{1}$ be a quasi-simple module of rank $r>1$ and $X_{i}=\tau^{-(i-1)} X_{1}$. Then there exist some $1 \leq j \leq r$ such that $\mu\left(X_{j}[r]\right) \geq \mu\left(H_{1}\right)$.

Proof. We first claim that for each indecomposable projective $\Lambda$-module $P$ with $\delta(P)=-1$, there exists an index $1 \leq j \leq r$ such that $\operatorname{Hom}\left(P, X_{j}[r-1]\right)=0$. If not, we take an indecomposable projective module $P_{t}$ with $\delta\left(P_{t}\right)=-1$ such that $\operatorname{dimHom}\left(P_{t}, X_{i}[r-1]\right)=\left(\underline{\operatorname{dim}} X_{i}[r-1]\right)_{t} \geq 1$ for all $1 \leq i \leq r$. Applying $\operatorname{Hom}\left(P_{t},-\right)$ to the short exact sequence $0 \rightarrow X_{2} \rightarrow X_{2}[r] \rightarrow X_{1}[r-1] \rightarrow 0$, we obtain that $\operatorname{dimHom}\left(P_{t}, X_{2}\right)=0$. Similarly, for each $1 \leq i \leq r,\left(\underline{\operatorname{dim}} X_{i}\right)_{t}=$ $\operatorname{dimHom}\left(P_{t}, X_{i}\right)=0$. This implies that $\delta_{t}=\sum_{i}\left(\underline{\operatorname{dim}} X_{i}\right)_{t}=0$, a contradiction.

Assume that $Y$ is a Gabriel-Roiter submodule of $H_{1}$. Then, by the Theorem, $Y$ is a preprojective module with defect $\delta(Y)=-1$, say $Y=\tau^{-m} P$ for some indecomposable projective module $P$ with $\delta(P)=-1$. Note that $\operatorname{Hom}\left(Y, X_{i}[r-\right.$ $1]) \cong \operatorname{Hom}\left(P, \tau^{m} X_{i}[r-1]\right) \cong \operatorname{Hom}\left(P, X_{s}[r-1]\right)$, where $m \equiv i-s(\bmod r)$. Since for $P$ there is an index $1 \leq j^{\prime} \leq r$ such that $\operatorname{Hom}\left(P, X_{j}^{\prime}[r-1]\right)=0$, we may get an index $j$ such that $\operatorname{Hom}\left(Y, X_{j}[r-1]\right)=0$. On the other hand, $\operatorname{Hom}\left(Y, X_{j}[r]\right) \neq 0$. If $f$ is a nonzero map in $\operatorname{Hom}\left(Y, X_{j}[r]\right)$, then the image is isomorphic to $Y$ or a regular submodule of $X_{j}[r]$ by Lemma 2.2 . However, $\operatorname{Hom}\left(Y, X_{j}[r-1]\right)=0$ and $|Y|<\left|H_{1}\right|=\left|X_{j}[r]\right|$ imply that $\operatorname{im} f$ cannot be regular. Thus $f$ is a monomorphism. It follows that $\mu\left(X_{j}[r]\right)>\mu(Y)$. If $\mu\left(X_{j}[r]\right)<\mu\left(H_{1}\right)$, then $\left|X_{j}[r]\right|>\left|H_{1}\right|$ by Lemma 2.1 since $Y$ is a GR submodule of $H_{1}$. This is a contradiction. Thus $\mu\left(X_{j}[r]\right) \geq \mu\left(H_{1}\right)$.

Corollary. Let $\Lambda=k Q$ be a tame hereditary algebra of type $\widetilde{\mathbb{A}}_{n}, \widetilde{\mathbb{D}}_{n}, \widetilde{\mathbb{E}}_{6}$ or $\widetilde{\mathbb{E}}_{7}$. Then each quasi-simple module $X$ is a Gabriel-Roiter factor and there are, up to isomorphism, infinitely many Gabriel-Roiter inclusions with factor $X$.

Proof. It is clear if $X \cong H_{1}$ is a simple homogeneous module by Proposition 2.4, Thus we may assume that the rank of $X$ is larger than 1.

Let $X=X_{1}=X_{1}[1]$ be a quasi-simple module of rank $r>1$. Write $X_{i}=$ $X_{i}[1]=\tau^{i-1} X_{1}$; then $X_{r+1} \cong X_{1}$ and in general $X_{s r+j} \cong X_{j}$ for each integer $s \geq 0$ and $1 \leq j \leq r$. There is a short exact sequence

$$
0 \rightarrow X_{i}[i-1] \rightarrow X_{i}[i] \rightarrow X_{1} \rightarrow 0
$$

for each $i \geq 2$. Assume that for a given integer $s>0$, none of these inclusions $X_{i}[i-1] \rightarrow X_{i}[i]$ are Gabriel-Roiter inclusions for $s r+1 \leq i \leq(s+1) r$. Then Proposition 2.5] implies that $\mu\left(X_{i}[r]\right)<\mu\left(H_{1}\right)$ for each $s r+1 \leq i \leq(s+1) r$. Since $X_{s r+j} \cong X_{j}$, we obtain that $\mu\left(X_{j}[r]\right)<\mu\left(H_{1}\right)$ for all $1 \leq j \leq r$, which is a contradiction. Therefore, for each given positive integer $s$ there is a Gabriel-Roiter inclusion $X_{i}[i-1] \subset X_{i}[i]$ with $s r+1 \leq i \leq(s+1) r$. We are done.

\section{ACKnowledgments}

The author is grateful to the anonymous referee(s), whose comments on Kronecker pairs substantially improved the results. He would also like to thank Jan Schröer for his hospitality during the author's stay in Bonn. 


\section{REFERENCES}

[1] B. Chen, The Gabriel-Roiter measure for representation-finite hereditary algebras. J. Algebra 309(2007), 292-317. MR2301241 (2007m:16013)

[2] B. Chen, The Auslander-Reiten sequences ending at Gabriel-Roiter factor modules over tame hereditary algebras. J. Algebra Appl. 6(2007), 951-963. MR2376793 (2008m:16035)

[3] B. Chen, The Gabriel-Roiter measure for $\widetilde{\mathbb{A}}_{n}$. J. Algebra 320(2008), 2891-2906. MR2442001 (2009h:16013)

[4] B. Chen, Comparison of Auslander-Reiten theory and Gabriel-Roiter measure approach to the module categories of tame hereditary algebras. Comm. Algebra 36(2008), 4186-4200. MR 2460409 (2009j:16018)

[5] V. Dlab; C. M. Ringel, Indecomposable representations of graphs and algebras. Mem. Amer. Math. Soc. 6(1976), no. 173. MR0447344(56:5657)

[6] D. Happel; C. M. Ringel, Tilted algebras. Trans. Amer. Math. Soc. 274(1982), 399-443. MR675063 (84d:16027)

[7] P. Gabriel, Indecomposable representations. II, Symposia Mathematica. Vol. XI, Academic Press, London, 1973, 81-104. MR0340377(49:5132)

[8] C. M. Ringel, The Gabriel-Roiter measure. Bull. Sci. Math. 129(2005), 726-748. MR2172139 (2006g:16039)

[9] C. M. Ringel, Foundation of the representation theory of Artin algebras, using the GabrielRoiter measure. Proceedings of the 36th Symposium on Ring Theory and Representation Theory, Vol. 2, 1-19, Symp. Ring Theory Represent. Theory Organ. Comm., Yamanashi, 2004. MR2077022 (2005g:16021)

Hausdorff Center for Mathematics, Universität Bonn, 53115 Bonn, Germany

Current address: Mathematisches Institut, Universität zu Köln, Weyertal 86-90, 50931 Köln, Germany

E-mail address: mcebbchen@googlemail.com 